\section{Rosacea: a clinical review}

\section{Carsten Sauer Mikkelsen, ${ }^{1}$ \\ Helene Ringe Holmgren, ${ }^{2}$ \\ Petra Kjellman, ${ }^{3}$ Michael Heidenheim, ${ }^{4}$ \\ Ari Kappinnen, ${ }^{5}$ Peter Bjerring, ${ }^{6}$ \\ Theis Huldt-Nystrom ${ }^{7}$ \\ ${ }^{1}$ Private Practitioner, Bronderslev, \\ Denmark; ${ }^{2}$ Private Practitioner, \\ Frederikshavn, Denmark; ${ }^{3}$ Private \\ Practitioner, Diagnostiskt Centrum Hud, \\ Stockholm, Sweden; ${ }^{4}$ Department of \\ Dermatology, Roskilde University \\ Hospital, Denmark; ${ }^{5}$ Private Practitioner, \\ Tampere, Finland; ' ${ }^{\text {Private Practitioner, }}$ \\ Molholm Private Hospital, Denmark; \\ ${ }^{7}$ Private Practitioner, Hudpoliklinikken, \\ Namsos, Norway}

\section{Abstract}

Rosacea is a field within dermatology with new insight within immunological research and new treatment-algorithm. Patient education on rosacea and appropriate treatments is an important aspect in helping patients succeed with therapy. Treatment should be tailored to each individual patient, taking into account: symptoms, trigger factors, patients' wishes, most bothersome symptoms, psychological aspect, individual needs. A combination of clinical therapies to treat different symptoms concomitantly may offer the best possible outcomes for the patient. In this review article we describe these aspects.

\section{Introduction}

Rosacea is a commonly encountered chronic inflammatory skin disease in adults with a predilection for highly visible areas of the skin such as the face. ${ }^{1}$ It is characterized by flushing, redness, pimples, pustules and dilated blood vessels. The eyes are often involved, and thickening of the skin with enlargement (phymas), especially of the nose, can occur in some people. ${ }^{2,3}$ Combination of symptoms and signs focused around the central face can be divided in primary and secondary features.

Although rosacea can occur in anyone, it most commonly affects middle-aged women with fair skin, blue eyes and blonde hair and less frequent in skin phototypes $\mathrm{V}$ and VI. Studies have shown up to $10 \%$ in the Swedish population and 2-3\% in France and Germany. Familial background is described in $15-40 \%$ of the patients. ${ }^{4}$ Three human leukocyte antigen - HLA alleles (MHC class II) are significantly associated with rosacea (HLA-DRB1, HLADQB1 and HLADQA1).

Because of the potential complexity of rosacea, it has been classified into the following 4 subtypes according to its signs and symptoms that often occur together: erythematotelangiectatic, papulopustular, phymatous and ocular.

\section{Erythemato-telangiectatic rosacea}

Erythemato-telangiectatic rosacea (ETR) is characterized by flushing and persistent redness of the central face, and often occurs before or at the same time as the bumps and pimples of subtype 2 (papulopustular) rosacea. Visible blood vessels may also be present. People with these signs of rosacea tend to have very sensitive skin, and may feel as if their skin stings or burns at times (Figure 1A).

\section{Papulopustular rosacea}

The symptoms of rosacea type 2 (papulopustular rosacea, PPR) may occur along with the facial redness and flushing of rosacea subtype 1 (Figure 1B).

Symptoms of rosacea subtype 2 includes: papules and/or pustules that come and go, combined with transient or persistent facial redness, primarily on the central face; burning and stinging; small visible blood vessels (telangiectasia); raised, scaly red patches known as plaques. While the papules and pustules of subtype 2 rosacea may resemble acne, there are generally no blackheads or whiteheads (comedones) present in rosacea alone. Acne more frequently affects also the back, shoulders, and chest.

This type of rosacea occurs most commonly in middle age and affects more commonly women than men.

\section{Rhinophyma (phymatous rosacea)}

Phymatous rosacea can affect nose (rhinophyma), chin (gnatophyma), forehead (metophyma), ears (otophyma) and eyelids (blepharophyma). Rhinophyma is the most frequent location shows marked skin thickenings and irregular surface nodularities especially of the nose. Telangiectasia can also be presented. Fibrosis is present and increased volume of sebaceous glands is observed.
Correspondence: Carsten Sauer Mikkelsen, Specialist in Dermato-Venereology, Private Practice, Bredgade 13, 2 sal, 9700 Brønderslev, Denmark.

Tel.: +45.201.00198 - Fax: +45.984.02424.

E-mail: c.s.mikkelsen@hotmail.com

Key words: Rosacea; erythema; papules; pustules; teleangiectasia; rhynophyma.

Contributions: CSM is the main author and contributor; the other authors contributed equally.

Conflict of interests: the authors declare no potential conflict of interest.

Key words: Rosacea; erythema; papules; pustules; teleangiectasia; rhynophyma.

Received for publication: 25 December 2015 Accepted for publication: 29 December 2015.

This work is licensed under a Creative Commons Attribution-NonCommercial 4.0 International License (CC BY-NC 4.0).

(C) Copyright C. Sauer Mikkelsen et al., 2016 Licensee PAGEPress, Italy

Dermatology Reports 2016; 8:6387

doi:10.4081/dr.2016.6387

Histopathological signs are characteristic dilated infundibulum and dense inflammatory infiltrate.

There are 4 histological types of rhinophyma that include glandular, fibrous, fibroangiomatous and actinic.

Rhinophyma was once thought to be caused by heavy alcohol use, but rhinophyma occurs equally in people who do not use alcohol and in those who drink heavily. The problem is much more common in men than in women (Figure 1C).

\section{Ocular rosacea}

Ocular rosacea range from minor irritation, foreign body sensation, dryness, and blurry vision to severe ocular surface disruption and inflammatory keratitis. Patients frequently describe a gritty feeling, and they commonly experience blepharitis and conjunctivitis. Other ocular findings include lid margin and conjunctival telangiectasias, eyelid thickening, eyelid crusts and scales, chalazion and hordeolum, punctate epithelial erosions, corneal infiltrates, corneal ulcers, corneal scars, and vascularization. Sight-threatening disease is rare with rosacea.

Ocular rosacea is most frequently diagnosed when patients also suffer from cutaneous disease. However, ocular signs and symptoms may occur prior to cutaneous man- 
ifestations in $20 \%$ of patients with rosacea. No correlation exists between the severity of ocular disease and the severity of facial rosacea (Figure 1D).

\section{Genetic profile}

Transcriptome analysis has shown a possible distinct gene profile for each subtype. ${ }^{6}$ More than $50 \%$ have a combination of the different subtypes. This is of importance since this patient group needs combination therapy for their rosacea.

\section{Psychological impact}

Rosacea can have a significant psychosocial impact on patients and cause anxiety, embarrassment and low self-esteem: ${ }^{7} 77 \%$ of patients are affected emotionally, $63 \%$ are affected at work, $67 \%$ are affected socially, $53 \%$ are affected in their relationships and dating behavior.

\section{Pathophysiology of rosacea}

The following implications associated with rosacea were investigated: i) vascular disease; ii) neurovascular component; iii) inflammation: innate immunity; iv) demodex folliculorum.

\section{A vascular disease}

Edema of the upper dermis leading to increased vascular permeability could be observed. Redness is frequent and confirmed most bothersome regardless of subtypes. ${ }^{8}$

There are many factors contributing to facial redness. Transient flushing may have variable intensity and frequency. Inflammatory lesions, lesional or perilesional redness, teleangiectasia, persistent macular background erythema are independent of lesions.

Inflammation from papules and pustules or dry inflamed skin may obscure the level of nontransient erythema.

Different treatments depend on type of erythema

\section{Neurovascular component}

The following is observed: increased skin sensitivity to noxious heat stimuli in rosaceaaffected skin, more prominent in those with PPR vs ETR; lower heat pain threshold in affected $v s$ non-affected areas (based on heating the skin with a probe, from $32^{\circ} \mathrm{C}$ to $50^{\circ} \mathrm{C}$ ); enhanced perception of noxious heat stimulus; subjective burning perception increased (based on VAS) in patients with rosacea $v s$ control subjects; elevated skin blood flow in PPR-affected skin $v s$ non-affected skin (based on LDI). This component is not significant for ETR-affected skin. ${ }^{9}$

The facial hypersensitivity is based on vascular changes due to: stasis, increased blood flow, inflammation, lower pain threshold (as described above), higher skin temperature and hypersensitivity (non-allergic). ${ }^{10}$

Neurogenic rosacea is a distinct clinical subtype requiring a modified approach to treatment with drugs like gabapentin, pregabalin and duloxetine. ${ }^{11}$

\section{Inflammation: innate and adaptive immunity}

Both the innate and adaptive immune system, are involved in the development of rosacea at a very early stage. Initially T-cells and macrophages infiltrate the skin, releasing factors leading to prolonged vasodilation, seen as erythema. They are also responsible for the recruitment of neutrophils and other cells, which later are clinically seen as pustules.

The critical cells involved in the inflammatory response in rosacea are the Th1 and Th17 cells, the mast cells, the macrophages, the antibody-presenting B cells, and the neutrophils.

Increased serine protease activity and cathelicidin promotes skin inflammation in rosacea. ${ }^{12}$

Mastcells are key mediators of cathelicidin- initiated skin inflammation in rosacea, ${ }^{13}$ but anti-histaminerg treatment is rarely effective in its treatment.

This knowledge of inflammation is, in our opinion, important to correct stepwise treatment-algorithm with initially treatment with anti-inflammatory drugs.

\section{Demodex}

Demodex are ubiquitous in the normal adult population, but not in neonates. It is considered as commensals with no clinical signs in most individuals. They are microscopic mites, commonplace all over the world with parasitic existence in hair follicles, sebaceous glands and eyelid glands. In immunocompromised people they may be found elsewhere. Demodex can be transmitted directly in areas rich in sebaceous glands, but also by indirect contaminated subjects such as towels, bed clothing etc. The life cycle is 14-18 days.

Two distinct species of Demodex mites are found in humans: Demodex folliculorum and D. brevis. ${ }^{14}$ The mites vary in size from $0.1 \mathrm{~mm}$ to $0.4 \mathrm{~mm}$ long.

A higher prevalence of $D$. folliculorum and mean mite density was found in rosacea patients especially with a higher density of mites in involved areas compared with controls, acne and LED. Demodex induces neutrophilic and granulomatous inflammation. Demodex in the eye can cause conjunctival inflammation, superficial corneal vascularization and scarring. ${ }^{15}$ Mite density increased sig-

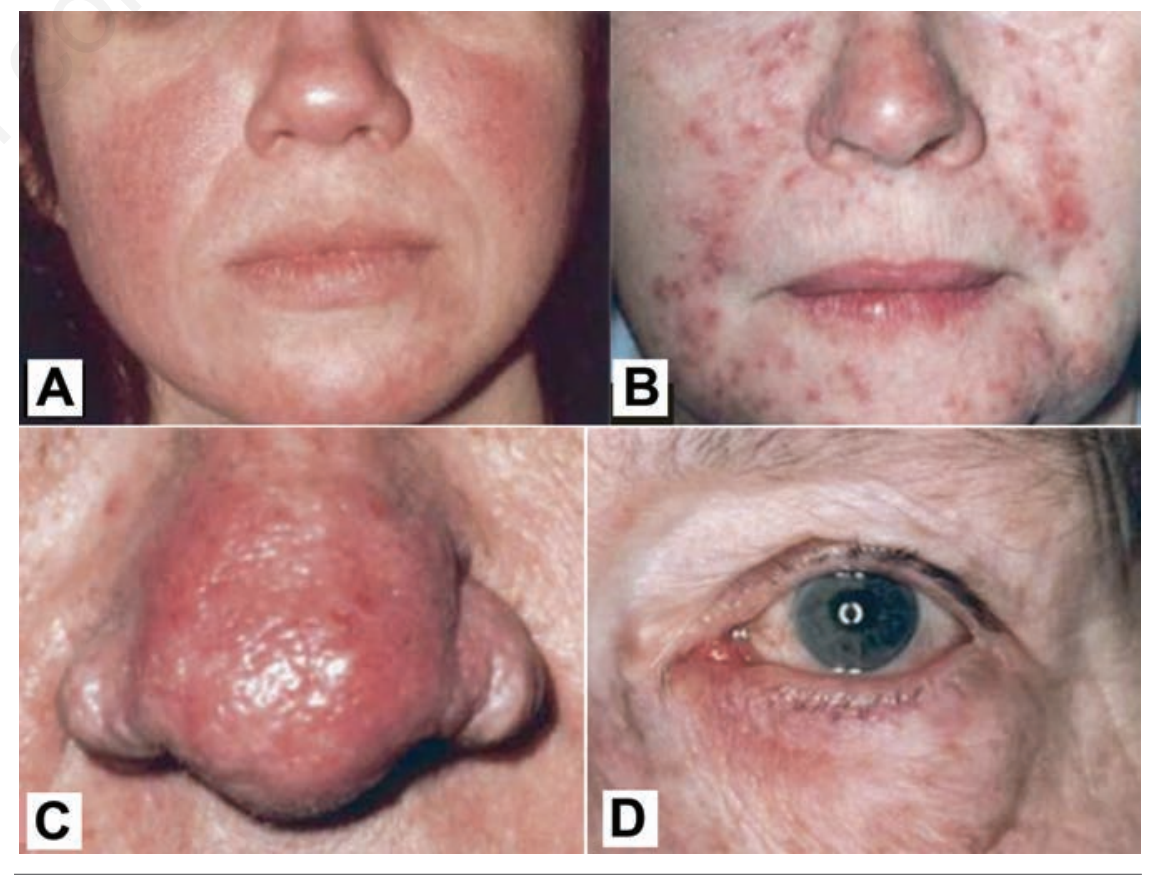

Figure 1. The 4 different types of rosacea examined: A) erythematotelangiectatic rosacea; B) papulopustular rosacea; C) rhinophyma (phymatous rosacea) and D) ocular rosacea. 
nificantly with the length of treatment with topical steroids $(\mathrm{P}<0.001){ }^{16}$

Neither doxycycline nor topical metronidazole $7.5-10 \mathrm{mg} / \mathrm{g}$ have a significant anti-parasitic effect. Demodex infestation does not decrease in parallel with improvement under tetracycline treatment.

Mite-related bacterial antigens such as $B$. oleronius sonicate might stimulate inflammatory cells in rosacea. Bacillus oleronius is a Gram-negative bacteria isolated in Demodex. ${ }^{17}$ The bacteria are sensitive to tetracyclines. There is a very complex microbiota in Demodex mites from rosacea patients. Pityriasis folliculorum (with spiky changes, discrete, fine whitish, partly yellowish small scales and papules) can be seen in the sebaceous hair follicles as a result of high density of $D$. folliculorum. If inflammation of the follicle occurs, a Rosacea-like condition is seen.

Mechanical blockage of hair follicle and sebaceous ducts increases number of mites and initial hyperkeratinization of the infundibulum and stagnation may promote bacterial overgrowth.

\section{Trigger avoidance}

Certain foods and beverages - spicy or caffeinated, and other triggers as sunlight, stress, strenuous exercise, some types of cosmetics, waterproof cosmetics, heavy foundations that require, makeup remover should be avoided. ${ }^{18}$

An UK-based study on 60,000 patients with rosacea $v s 60,000$ controls showed a lower risk of rosacea in smokers: OR: 0.65 (95\%CI: 0.62$0.67)=$ protective. $^{19}$

\section{Photo protection in rosacea}

UV light is dangerous for rosacea since it stimulates innate inflammation; that's the reason why photo protection should be integrated into rosacea management (at least SPF 30). Unfortunately rosacea patients are susceptible to irritation caused by sunscreen ingredients. Appropriate protective ingredients (dimethicone, cyclomethicone) in the vehicle can minimize irritation. Physical, inorganic sun blocks (titanium, zinc oxide) are usually well tolerated. Newer products utilizing micro fine particle are under evaluation. ${ }^{20}$

\section{Make-up in rosacea}

Selecting appropriate cosmetics is essential. Waterproof and opaque make-up is preferred. A make-up containing ingredient that provides sun protection and decrease inflammation is recommended. Make-up with lower allergenic potential should be used. Mineral make-up is well tolerated. Formulations containing silica and talc are usually used. The aim is to give a matte finish to the complexion. ${ }^{21}$

\section{Treatment}

Patient education on rosacea and appropriate treatments is an important aspect in helping patients succeed with therapy. ${ }^{22}$ Treatment should be tailored to each individual patient, taking into account: symptoms, trigger factors, patients' wishes: most bothersome symptoms; psychological aspect; individual needs.

A combination of clinical therapies to treat different symptoms concomitantly may offer the best possible outcomes for the patient.

Erythema was universally reported as the most bothersome feature of rosacea in a study by Tan et al. ( $82 \%$ of 135 subjects).

\section{Erythema}

It is important to differentiate the underlying erythema, as its management will differ. Lesional/perilesional erythema can resolve with successful anti-inflammatory treatment. Telangiectatic rosacea is amenable to lasers /IPL management. ${ }^{23,24}$ To treat persistent macular background erythema, adrenergic receptor agonists can be used.

\section{Treatment considerations (chronologically based)}

\section{Papulopustular rosacea}

Our suggestion for initial treatment would be to use a highly well tolerated topical or an oral anti-inflammatory agent first. Topical ivermectin, which appears to have less cutaneous irritation than the metronidazole products, may be a reasonable first choice. In patients who have more inflammatory lesions, we would probably opt for the addition of a cyclin such as a subantimicrobial-dose doxycycline.

In our experience it's important to treat the inflammatory activity first and typically for about 4 weeks before treating the teleangiectasia or erythema. Isotretinoin $0.3 \mathrm{mg} / \mathrm{kg}$ is effective for the widespread inflammatory lesion.

\section{Dye-laser therapy}

Dye-laser therapy is very beneficial for the treatment of teleangiectasia. However, if you apply it too early in very inflamed skin, it can lead to stinging and burning. If we first get the inflammation under control with a systemic drug in combination with a topical, and then perform the laser therapy later, these side effects occurs much more rarely. After the telangiectasia was well treated with laser only the background erythema is left.

\section{Brimonidine}

In the end, brimonidine could be used to minimize the background erythema, but only after the inflammatory lesions and teleangiectasias are gone.

\section{A stepwise approach}

A stepwise approach therefore includes: i) getting the inflammatory lesions under control; ii) using laser modality to get rid of the blood vessels (under control), iii) and finally use brimonidine to (just) minimize the background erythema (Figure 2).

Cochrane database helps professionals and patients with evidence-informed health decision-making by producing high-quality, relevant systematic review.

GRADE (Grading of Recommendations,

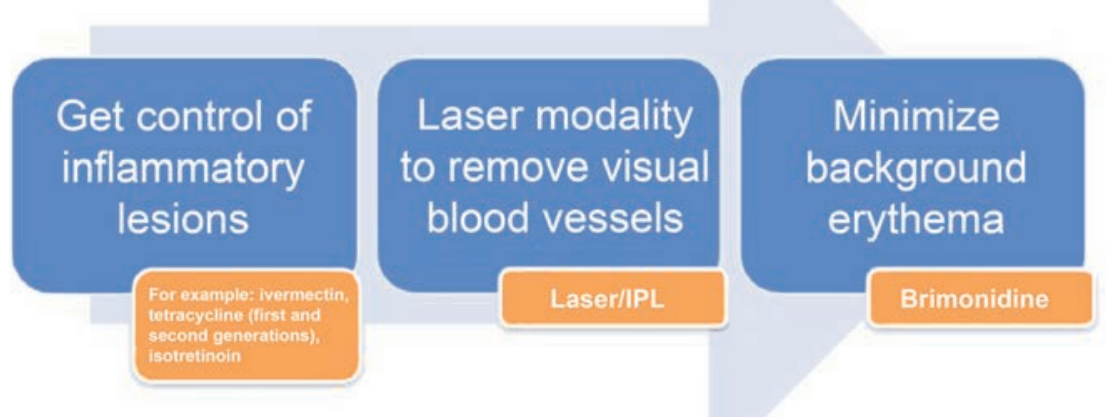

Figure 2. Stepwise treatment of rosacea. 
Table 1. Clinical description of rosacea, according to the GRADE classification.

\begin{tabular}{lcl} 
GRADE & Score & Clinical description \\
Clear & 0 & No inflammatory lesions present, no erythema \\
Almost clear & 1 & Very few small papules/pustules, very mild erythema present \\
\hline Mild & 2 & Few small papules/pustules, mild erythema \\
Moderate & 3 & Several small or large papules/pustules, moderate erythema \\
\hline Severe & 4 & Numerous small and/or large papules/pustules, severe erythema \\
\hline
\end{tabular}

Assessment, Development and Evaluation) scale helps in making judgments about quality of evidence and strength of recommendations (Table 1).

\section{Primarily anti-inflammatory drugs}

High-quality evidence for all outcomes, supports that ivermectin is effective and safe for papulopustular rosacea in two trials in 1371 participants..$^{25}$ High-quality evidence supports that ivermectin was more effective than metronidazole in papulopustular rosacea one trial in 962 participants. ${ }^{26}$ Modified-release doxycycline $40 \mathrm{mg}$ might be as effective as doxycycline $100 \mathrm{mg}$ (quality of the evidence: low), with a quarter of the side effects. Azithromycin might be as effective as doxycycline $100 \mathrm{mg}$ (quality of the evidence: very low). Isotretinoin $0.3 \mathrm{mg} / \mathrm{kg}$ versus doxycycline $100 \mathrm{mg}$ (after 2 weeks, tapered to $50 \mathrm{mg} /$ day). High-quality evidence for all outcomes supporting that isotretinoin was more effective than doxycycline in papulopustular rosacea one trial in 262 participants. ${ }^{27}$

\section{Treatment of telangiectasia}

Nine randomized controlled trials with dye and laser and/or light-based therapies appeared to be effective, but limited data are provided and small sample sizes are considered. Effects on telangiectasia and to a lesser extent on erythema are of low quality of evidence. . $^{28-32}$

Primarily against erythema, brimonidine is a highly selective 2-adrenergic receptor agonist with vasoconstrictive activity. It starts working within 30 minutes and works up to 12 hours. There is high-quality evidence for all outcomes, supporting that brimonidine is twice as effective as vehicle for erythema. In rosacea it is the first and only proven efficacious treatment for persistent erythema. ${ }^{33}$

Lastly, we still need more RCTs for recommendation on treatment of ocular rosacea.

\section{Conclusions}

Targeting symptoms and signs with novel treatments (stepwise) and combinations of therapy will significantly improve rosacea. Clear instructions and expectations are required to optimize therapy. Advice on cosmetics, camouflage and photo protection, as well as avoiding triggers, should be part of any consultation.

\section{References}

1. Lanoue J, Goldenberg G. Therapies to improve the cosmetic symptoms of rosacea. Cutis 2015;96:19-26

2. Van Zuuren EJ, Fedorowicz Z, Carter B et al. Interventions for rosacea. Cochrane Database Syst Rev 2015;4:CD003262.

3. Wilkin J, Dahl M, Detmar M, et al. Standard classification of rosacea: report of the National Rosacea Society Expert Committee on the classification and staging of rosacea. $\mathrm{J}$ Am Acad Dermatol 2002;46:584-7.

4. Abram K, Silm H, Maaroos HI, et al. Risk factors associated with rosacea. J Eur Acad Dermatol Venereol 2010;24:565-71.

5. Chang AL, Raber I, Xu J, et al. Assessment of the genetic basis of rosacea by genomewide association study. J Invest Dermatol 2015;135:1548-55.

6. Steinhoff M, Buddenkotte J, Aubert J, et al. Clinical, cellular, and molecular aspects in the pathophysiology of rosacea. J Investig Dermatol Symp Proc 2011;15:2-11.

7. Dirschka T, Micali G, Papadopoulos L, et al. Perceptions on the psychological impact of facial erythema associated with rosacea: results of international survey. Dermal There (Heidelb) 2015;5:117-27.

8. Tan J, Blume-Peytavi U, Ortonne JP, et al. An observational cross-sectional survey of rosacea: clinical associations and progression between subtypes. Br J Dermatol 2013;169:555-62.

9. Guzman Sanchez DA, Ishiuji Y, Patel T et al. Enhanced skin blood flow and sensitivity to noxious heat stimuli in papulopustular rosacea. J Am Acad Dermatol 2007;57:800-5.

10. Lohne-Rahm S, Nordlind K, Berg M, et al.
Laser treatment of rosacea: a pathoetiological study. Arch Dermatol 2004;140:1345-9.

11. Scharschmidt TC, Yost JM, Truong SV, et al. Neurogenic rosacea: a distinct clinical subtype requiring a modified approach to treatment. Arch Dermatol 2011;147:123-6.

12. Yamasaki K, Di Nardo A, Bardan A, et al. Increased serine protease activity and cathelicidin promotes skin inflammation in rosacea. Nat Med 2007;13:975-80.

13. Muto Y, Wang Z, Vanderberghe M, et al. Mast cells are key mediators of cathelicidin-initiated skin inflammation in rosacea. J Invest Dermatol 2014;134:272836 .

14. Lacey N, Forton FM, Powell FC. Demodex quantification methods: limitations of confocal laser scanning microscopy. Br J Dermatol 2013;169:212-3.

15. Kheirkhah A, Casas V, Li W, et al. Corneal manifestations of ocular demodex infestation. Am J Ophthalmol 2007;143:743-9.

16. Dolenc-Voljc M, Pohar M, Lunder T. Density of Demodex folliculorum in perioral dermatitis. Acta Derm Venereol 2005;85:211-5.

17. Szkaradkiewicz A, Chudzicka-Strugata I, Karpinski TM, et al. Bacillus oleronius and Demodex mite infestation in patients with chronic blepharitis. Clin Microbiol Infect 2012;18:1020-5.

18. Van Zuuren EJ, Fedorowicz Z. Interventions for rosacea: abridged updated Cochrane review including GRADE assessments. Br J Dermatol 2015;173:65162.

19. Spoendlin J, Voegel JJ, Jick SS, Meier CR. A study on the epidemiology of rosacea in the U.K. Br J Dermatol 2012;167:598-60.

20. Eleweski BE, Draelos Z, Dreno B et al. Rosacea - global diversity and optimized outcome: proposed international consensus from the Rosacea International Expert Group. J Eur Acad Dermatol Venereol 2011;25:188-200.

21. Levy LL, Emer JJ. Emotional benefit of cosmetic camouflage in the treatment of facial skin conditions: personal experience and review. Clin Cosmet Investing Dermatol 2012;5:173-82.

22. Tanghetti EA, Jackson JM, Belasco KT, et al. Optimizing the use of topical brimonidine in rosacea management: panel recommendations. J Drugs Dermatol 2015;1 4:33-40.

23. Nymann P, Hedelund L, Haedersdal M. Long-pulsed dye laser vs intense pulsed light for the treatment of facial teleangiectasias: a randomized controlled trial. J Eur Acad Dermatol Venereol 2010;24:143-6.

24. Papageorgiou P, Clayton W, Norwood S, et 
al. Treatment of rosacea with intense pulsed light: significant improvement and long-lasting results. $\mathrm{Br} \mathrm{J}$ Dermatol 2008;159:628-32.

25. Stein L, Kircik L, Fowler J et al. Efficacy and safety of ivermectin $1 \%$ cream in treatment of papulopustular rosacea: results of two randomized, double-blind, vehicle-controlled pivotal studies. J Drugs Derm 2014;13:316-23.

26. Taieb A, Ortonne JP, Ruzicka T, et al. Superiority of ivermectin $1 \%$ cream over metronidazole $0.75 \%$ cream in treating inflammatory lesions of rosacea: a randomized, investigator blinded trial. Br J Dermatol 2015;172:1103-10.

27. Gollnick H, Blume-Peytavi U, Szabo EL, et al. Systemic isotretinoin in the treatment of rosacea - doxycycline - and placebo-controlled, randomized clinical study. J Dtsch Dermatol Ges 2010;8:505-15.

28. Alam M, Voravutinon N, Warycha M, et al. Comparative effectiveness of non purpuragenic 595-nm pulsed dye laser and microsecond $1064 \mathrm{~nm}$ neodymium: yttrium-aluminium-garnet laser for treatment of diffuse facial erythema: a double-blind randomized controlled trial. J Am Acad Dermatol 2013;69:438-43.

29. Kim TG, Oh YS, Ji JH et al. Rosacea (erythematoteleangiectatic type) effectively improved by topical xylometazoline. Br J Dermatol 2011;38:510-3.

30. Neuhaus IM, Zane LT, Tope WD, et al. Comparative efficacy of nonpurpuragenic pulsed dye laser and intense pulsed light for erythematoteleangiectatic rosacea. Dermatol Surg 2009;35:920-8.

31. Nymann P, Hedelund L, Haedersdal M. Long-pulsed dye laser vs intense pulsed light for the treatment of facial teleangiectasies: a randomized controlled trial. J Eur Acad Dermatol Venereol 2010;24:143-9.

32. Fowler J Jr, Jackson M, Moore A, et al. Efficacy and safety of once-daily topical brimonidine tartrate gel $0.5 \%$ for the treatment of moderate to severe facial erythema of rosacea: results of two randomized, double blinded and vehicle-controlled pivotal studies. J Drugs Dermatol 2013;12:650-6. 